2. To: (Receiving Organization)

Consequence Analysis (8M400)

5. Proj./Prog./Dept./Div.:

TWRS FSAR/BIO

8. Originator Remarks:

The attached SD documents the originator's analysis on $7 y$. It shall not be used as the final or sole document for effecting changes to an authorization basis or safety basis for a facility or activity.

11. Receiver Remarks:
3. From: (originating organization)

6. Cog. Engr.:

R. J. Van Vleet
Consequence Analysis (8M400)
4. Related EDT No.:

NA
- thase Order No.

NA

9. Equip./Comoonent Mo.:

NA

10. Systen/8Idg./Facility:

Single-Shell Tank:

Double-Shell Tanks

Double-Containod Receiver Tanks

12. Major Assm. Dwg. No.:

NA

13. Permit/Permit Application No.: NA

14. Required Response Date:

NA

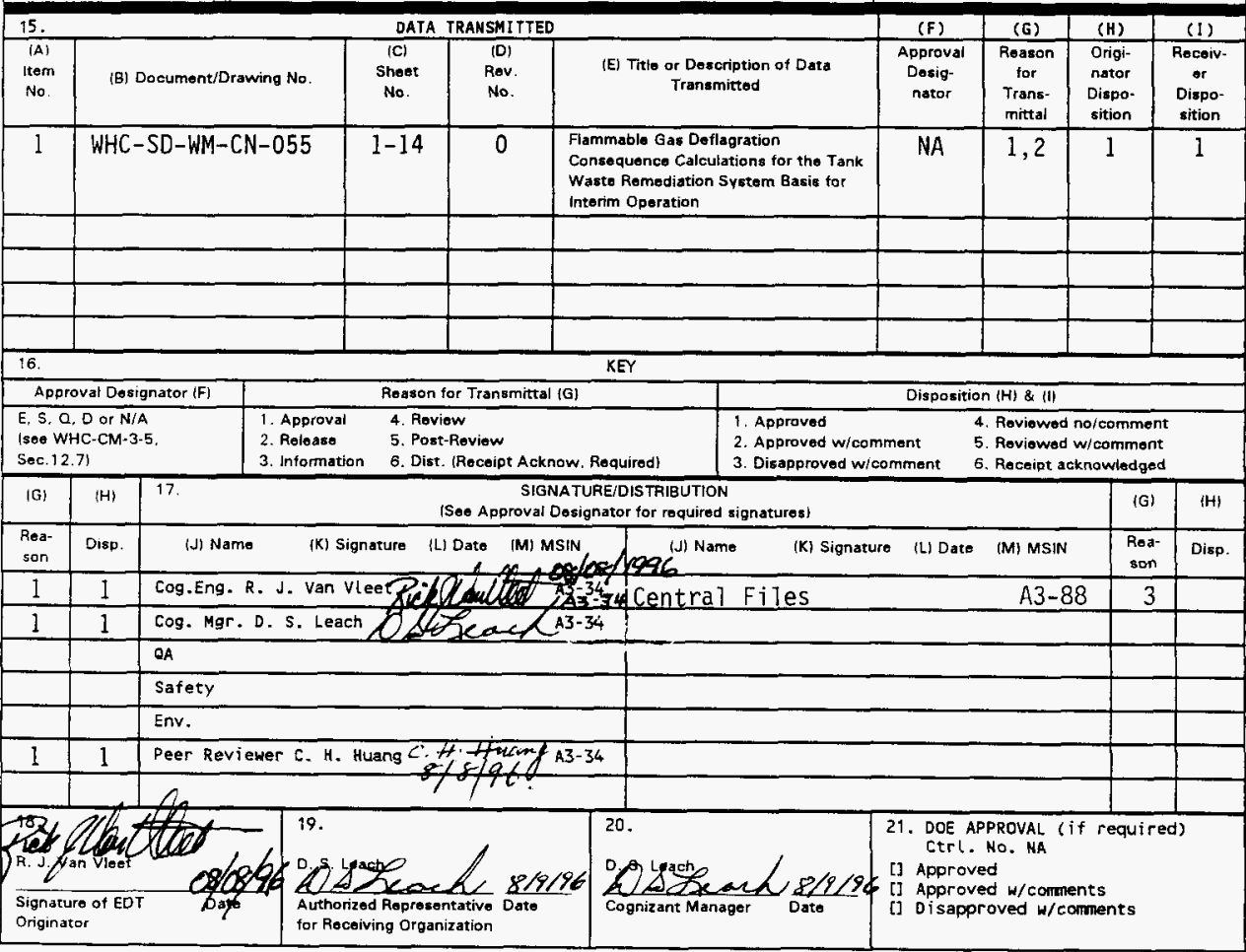

BD-7400-172-2(04/94) GEF097 


\title{
Flammable Gas Deflagration Consequence Calculations for the Tank Waste Remediation System Basis for Interim Operation
}

\author{
R. J. Van V1eet
}

Westinghouse Hanford Company, Richland, WA 99352

U.S. Department of Energy Contract DE-AC06-87RL10930

\begin{tabular}{|c|c|c|}
\hline $\begin{array}{l}\text { EDT/ECN: } \\
\text { Org Code } \\
\text { B\&R Code }\end{array}$ & $\begin{array}{l}614541 \\
8 M 400 \\
\text { EW3120071 }\end{array}$ & $\begin{array}{l}\text { UC: } 2030 \\
\text { Charge Code: } \\
\text { Total Pages: }\end{array}$ \\
\hline
\end{tabular}

Key Words: deflagrations, toxic exposures, dose consequences, singleshell tanks, double-shell tanks, double-contained receiver tanks, catch tanks

Abstract: This paper calculates the radiological dose consequences and the toxic exposures for deflagration accidents at various Tank Waste Remediation System facilities. These will be used in support of the Tank Waste Remediation System Basis for Interim Operation.

TRADEMARK DISCLAIMER. Reference herein to any specific comercial product, process, or service by trade name, tradenark, manufacturer, or otherwise, does not necessarily constitute or imply its endorsement, recommendation, or favoring by the United States Government or any agency thereof or its contractors or subcontractors.

Printed in the United States of America. To obtain copies of this document, contact: WHC/BCS Document Control Services, P.O. Box 1970, Mailstop H6-08, Richland WA 99352, Phone (509) 372-2420; Fax (509) 376-4989.
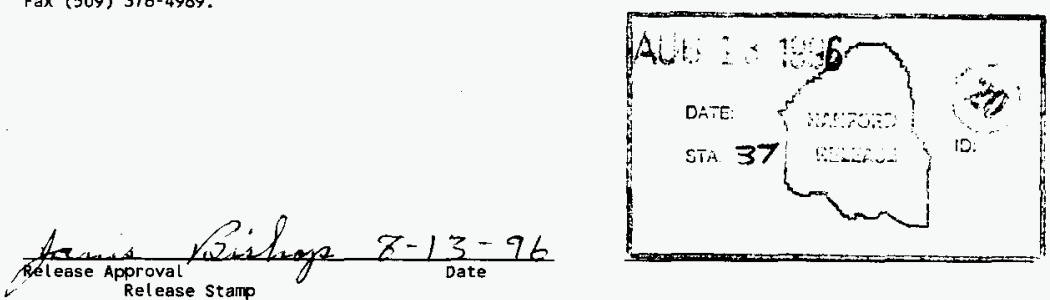

\section{Approved for Public Release}


WHC-SD-WM-CN-055, Rev. O

This page intentionally left blank. 


\section{FLAMMABLE GAS DEFLAGRATION CONSEQUENCE CALCULATIONS FOR THE TANK WASTE REMEDIATION SYSTEM BASIS FOR INTERIM OPERATION}

This calculation note calculates the radiological consequences and toxicological exposures associated with various deflagration accident scenarios. The radiological consequences were calculated using the unit liter doses found in Cowley (1996) and the methodology for calculating consequences is found in Van Keuren and Savino (1996). The unit liter doses are the radiation doses received per liter of tank waste. The doses were calculated using the pathways of submersion (direct radiation), inhalation, and 24-hour ingestion. Cowley (1996) provides the technical basis for the unit liter doses. Van Keuren and Savino (1996) states that the following equation is to be used for calculating the inhalation and submersion dose. Note: generally, the submersion dose is insignificant (orders of magnitude lower) compared to the inhalation dose.

$$
E D E_{\text {inhalation }}=Q \frac{\chi}{Q^{\prime}} B_{r} U L D_{\text {inhalation }}
$$

where $Q$ is the volume of waste resuspended $(L), X / Q^{\prime}$ is the atmospheric dispersion coefficient $\left(\mathrm{s} / \mathrm{m}^{3}\right), B_{r}$ is the standard man breathing rate during light activity $\left(\mathrm{m}^{3} / \mathrm{s}\right)$, and the ULD $D_{\text {inhalation }}$ is the unit liter dose $(\mathrm{S} v / L)$ due to inhalation and submersion.

Ingestion doses are calculated using the following equation.

$$
E D E_{\text {ingestion }}=\varrho \frac{\chi}{Q^{\prime}} U L D_{\text {ingestion }}
$$

where again, $Q$ is the volume of waste resuspended (L), $X / Q^{\prime}$ is the atmospheric dispersion coefficient $\left(\mathrm{s} / \mathrm{m}^{3}\right)$ and ULD ingestion is the unit liter dose $\left(\mathrm{Sv} \cdot \mathrm{m}^{3} / \mathrm{s} \cdot \mathrm{L}\right)$ due to 24-hour ingestion.

The toxicological consequences were calculated using the sum-offractions values and methodology found in Van Keuren (1996). For a release of solid or liquid toxic materials, the peak concentration is of concern. The following equation was used to calculate the peak concentration, $\mathrm{C}\left(\mathrm{mg} / \mathrm{m}^{3}\right)$ for a continuous release of solid or liquid toxic material. 


$$
C_{i}=Q^{\prime} \frac{X}{Q^{\prime}}
$$

where $C_{i}$ is the concentration of the $i^{\text {th }}$ toxic material, $Q^{\prime}$ is the release rate (mg/s) and $X / Q^{\prime}$ is the integrated atmospheric dispersion coefficient $\left(\mathrm{s} / \mathrm{m}^{3}\right)$.

This method has been extended to include the multiple chemicals contained in the tank waste. To do this, a sum-of-fractions methodology has been applied. That is, on a per unit basis, the ratio of a chemicals concentration (calculated either at the $100 \mathrm{~m}$ receptor or the offsite receptor) is compared to the appropriate limit. This was done for all chemicals involved, then the ratios or fractions were summed.

$$
\text { SOF }=\frac{C_{1}}{\text { Limit }}+\frac{C_{2}}{\text { Limit }_{2}}+\ldots+\frac{C_{n}}{L i m i t_{n}}
$$

If the sum-of-fractions is less than or equal to 1 , the risk guidelines are considered to have been met. If the sum-of-fractions is greater than 1 , the risk guidelines are considered to have been exceeded. Thus, the above equation now becomes,

$$
T=Q^{\prime} S O F
$$

where $T$ is the measure of acceptance, $Q^{\prime}$ is the toxic material release rate $(L / s)$ and SOF is the sum-of-fractions of the risk guidelines $(s / L)$ for either the onsite or offsite receptor. For a puff release, $Q^{\prime}$ is the amount of toxic material released (L) and SOF is the sum-offractions of the risk guidelines $(1 / L)$. The sum-of-fractions for each accident has been calculated for each of the frequency categories, i.e., anticipated, unlikely, and extremely unlikely. The following spreadsheet pages document the calculations. The maximum waste material accumulation in ventilation systems is taken from WHC-SD-WM-CN-054, Rev. 0 (Van Vleet 1996). An in-depth example calculation is shown here for the single-shell tanks. Other deflagration accidents in Tank Waste Remediation System facilities are treated in a similar fashion.

\section{EXAMPLE}

\section{Radiological Dose Consequences - Onsite}

The radiological dose consequences from a deflagration will consist of the sum of two releases. These releases are (1) the matepial from the ventilation system and (2) the material entrained from the tank waste by the deflagration. The following paragraphs show how the values where calculated for each release. 
The cumulative effective dose equivalent for the onsite receptor from the calculated release of waste accumulations in the ventilation system for a single-shell tank due to a high pressure event (such as a deflagration) is

$$
\begin{aligned}
E D E_{\text {inhalation }} & =Q \frac{\chi}{Q^{\prime}} B_{r} U L D_{\text {inhalation }} \\
& =(0.00613 \mathrm{~L})\left(3.41 \times 10^{-2} \frac{\mathrm{s}}{\mathrm{m}^{3}}\right)\left(3.3 \times 10^{-4} \frac{\mathrm{m}^{3}}{\mathrm{~s}}\right)\left(2.2 \times 10^{5} \frac{\mathrm{SV}}{\mathrm{L}}\right) \\
& =1.517 \times 10^{-2} \mathrm{SV}
\end{aligned}
$$

where the material released is $0.00613 \mathrm{~L}$ (Van Vleet 1996), the acute atmospheric dispersion coefficient is $3.41 \times 10^{-2} \mathrm{~s} / \mathrm{m}^{3}$ (Van Keuren and Savino 1996), the breathing rate for light activity is $3.3 \times 10^{-4} \mathrm{~m}^{3} / \mathrm{s}$ (Van Keuren and Savino 1996), and the ULD for single-shell tank solids is $2.2 \times 10^{5} \mathrm{~Sv} / \mathrm{L}$ (Cowley 1996).

The amount of material entrained from the waste surface is a combination of the material in the headspace (that natura11y in the headspace and that introduced by the gas release event) and the material entrained by the flame front (LANL 1996).

$$
\begin{aligned}
M_{\text {deflagration }} & =M_{\text {headspace }}+M_{\text {entrained }} \\
& =0.35 \mathrm{~kg}+5.45 \mathrm{~kg} \\
& =5.80 \mathrm{~kg}
\end{aligned}
$$

This material is assumed to be $100 \%$ respirable material (LANL 1996). Only a portion of this material will exit the tank. The fraction released can be calculated based upon the pressure the tank reaches and atmospheric pressure.

$$
\begin{aligned}
M_{\text {released }} & =(5.8 \mathrm{~kg})\left(\frac{(4 \mathrm{bar})\left(100000 \frac{\mathrm{Pa}}{\mathrm{bar}}\right)-101325 \mathrm{~Pa}}{(4 \mathrm{bar})\left(100000 \frac{\mathrm{Pa}}{\mathrm{bar}}\right)}\right) \\
& =(5.8 \mathrm{~kg})(0.747) \\
& =4.33 \mathrm{~kg}
\end{aligned}
$$


Using an assumed density of $1.65 \mathrm{~kg} / \mathrm{L}$, the volume of material released due to the deflagration is

$$
\begin{aligned}
Q & =\frac{4.33 \mathrm{~kg}}{1.65 \frac{\mathrm{kg}}{\mathrm{L}}} \\
& =2.62 \mathrm{~L}
\end{aligned}
$$

The cumulative effective dose equivalent to the onsite receptor from the calculated release of material during a deflagration is

$$
\begin{aligned}
E D E_{\text {inhalation }} & =\rho \frac{\chi}{\ell^{\prime}} B_{Y} U L D_{\text {Inhalation }} \\
& =(2.62 \mathrm{~L})\left(3.41 \times 10^{-2} \frac{\mathrm{s}}{\mathrm{m}^{3}}\right)\left(3.3 \times 10^{-4} \frac{\mathrm{m}^{3}}{\mathrm{~s}}\right)\left(2.2 \times 10^{5} \frac{\mathrm{SV}}{\mathrm{L}}\right) \\
& =6.5 \mathrm{SV}
\end{aligned}
$$

where the material released is $2.62 \mathrm{~L}$, the acute atmospheric dispersion coefficient is $3.41 \times 10^{-2} \mathrm{~s} / \mathrm{m}^{3}$ (Van Keuren and Savino 1996), the breathing rate is $3.3 \times 10^{-4} \mathrm{~m}^{3} / \mathrm{s}$ (Van Keuren and Savino 1996), and the ULD for single-shell tank solids is $2.2 \times 10^{5} \mathrm{~Sv} / \mathrm{L}$ (Van Keuren and Savino 1996).

Thus, the total cumulative effective dose equivalent to the onsite receptor is

$$
\begin{aligned}
C E D E & =C E D E_{\text {ventilation }}+C E D E_{\text {deflagration }} \\
& =1.517 \times 10^{-2} \mathrm{SV}+6.5 \mathrm{SV} \\
& =6.5 \mathrm{SV}
\end{aligned}
$$

\section{Radiological Dose Consequences - Offsite}

The cumulative effective dose equivalent for the offsite receptor from inhalation of the calculated release of waste accumulations in the ventilation system for a single-shell tank due to a high pressure event (such as a deflagration) is

$$
\begin{aligned}
E D E_{\text {inhalation }} & =\varrho \frac{\chi}{Q^{\prime}} B_{Y} U L D_{\text {inhalation }} \\
& =(0.00613 \mathrm{~L})\left(2.83 \times 10^{-5} \frac{\mathrm{s}}{\mathrm{m}^{3}}\right)\left(3.3 \times 10^{-4} \frac{\mathrm{m}^{3}}{\mathrm{~s}}\right)\left(2.2 \times 10^{5} \frac{\mathrm{SV}}{\mathrm{L}}\right) \\
& =1.259 \times 10^{-5} \mathrm{SV}
\end{aligned}
$$

where the material released is $0.00613 \mathrm{~L}$ (Van Vleet 1995), the acute atmospheric dispersion coefficient is $2.83 \times 10^{-5} \mathrm{~s} / \mathrm{m}^{3}$ (Van Keuren and 
Savino 1996), the breathing rate is $3.3 \times 10^{-4} \mathrm{~m}^{3} / \mathrm{s}$ (Van Keuren and Savino 1996), and the ULD for single-shel1 tank solids is $2.2 \times 10^{5} \mathrm{~Sv} / \mathrm{L}$ (Cowley 1996).

The cumulative effective dose equivalent to the offsite receptor from inhalation of the calculated release of material during a deflagration is

$$
\begin{aligned}
E D E_{\text {Inhalation }} & =\varrho \frac{\chi}{Q^{\prime}} B_{r} U L D_{\text {inhalation }} \\
& =(2.62 \mathrm{~L})\left(2.83 \times 10^{-5} \frac{\mathrm{s}}{\mathrm{m}^{3}}\right)\left(3.3 \times 10^{-4} \frac{\mathrm{m}^{3}}{\mathrm{~s}}\right)\left(2.2 \times 10^{5} \frac{\mathrm{SV}}{\mathrm{L}}\right) \\
& =5.383 \times 10^{-3} \mathrm{SV}
\end{aligned}
$$

where the material released is $2.62 \mathrm{~L}$, the acute atmospheric dispersion coefficient is $2.83 \times 10^{-5} \mathrm{~s} / \mathrm{m}^{3}$ (Van Keuren and Savino 1996), the breathing rate for 1 ight activity is $3.3 \times 10^{-4} \mathrm{~m}^{3} / \mathrm{s}$ (Van Keuren and Savino 1996), and the ULD for single-shell tank solids is $2.2 \times 10^{5} \mathrm{~Sv} / \mathrm{L}$ (Cowley 1996).

The cumulative effective dose equivalent to the offsite receptor due to 24-hour ingestion is

$$
\begin{aligned}
C E D E_{\text {ingestion }} & =\varrho \frac{\chi}{Q^{\prime}} U L D_{\text {ingestion }} \\
& =(0.00613 \mathrm{~L}+2.62 \mathrm{~L})\left(2.83 \times 10^{-5} \frac{\mathrm{s}}{\mathrm{m}^{3}}\right)\left(4.10 \frac{\mathrm{SV} \mathrm{m}}{\mathrm{s}}\right) \\
& =3.05 \times 10^{-4} \mathrm{SV}
\end{aligned}
$$

Thus, the total cumulative effective dose equivalent to the offsite receptor is

$$
\begin{aligned}
C E D E & =C E D E_{\text {ventilation }}+C E D E_{\text {deflagration }}+C E D E_{\text {ingestion }} \\
& =1.26 \times 10^{-5} \mathrm{SV}+5.39 \times 10^{-3} \mathrm{SV}+3.05 \times 10^{-4} \mathrm{SV} \\
& =5.7 \times 10^{-3} \mathrm{SV}
\end{aligned}
$$




\section{Toxicological Exposures - Onsite}

The sum-of-fractions for each accident has been calculated for each of the frequency categories, i.e., anticipated, unlikely, and extremely unlikely. This example demonstrates the method for anticipated, continuous releases. Calculations for other frequencies or for puff releases are similar.

Toxic exposures were calculated from three sources: (1) material released from the ventilation system, (2) material released from the tank due to the deflagration, and (3) pre-existing (i.e., before the deflagration) material released from the headspace.

For material released from the ventilation system, the onsite sum-offractions is:

$$
\begin{aligned}
T_{\text {ventilation }} & =Q^{\prime} S O F \\
& =\left(\frac{0.00613 L}{200 \mathrm{~S}}\right)\left(40000 \frac{\mathrm{S}}{L}\right) \\
& =1.23
\end{aligned}
$$

where $200 \mathrm{~s}$ is the duration of the release. The release duration was chosen because the burn is over in approximately 1/10 s (LANL 1995), the pressure pulse is over in approximately $10 \mathrm{~s}$ (LANL 1995), then the release continues because of temperature of the tank is still high.

Similarly for the material released from the deflagration.

$$
\begin{aligned}
T_{\text {deflagration }} & =Q^{\prime} S O F \\
& =\left(\frac{2.62 L}{200 \mathrm{~S}}\right)\left(40000 \frac{\mathrm{S}}{L}\right) \\
& =524
\end{aligned}
$$

For the material pre-existing in the headspace, the onsite sum-offractions is:

$$
\begin{aligned}
T_{\text {headspace }} & =Q^{\prime} \text { SOF } \\
& =\left(\frac{3350 \mathrm{~m}^{3}}{200 \mathrm{~s}}\right)\left(77 \frac{\mathrm{S}}{\mathrm{m}^{3}}\right) \\
& =1290
\end{aligned}
$$

where $3350 \mathrm{~m}^{3}$ represents the volume of the headspace in single-shell tank 241-A-103. Thus, the total toxicological exposure to the onsite receptor is

$$
\begin{aligned}
T & =T_{\text {ventilation }}+T_{\text {deflagration }}+T_{\text {headspace }} \\
& =1.23+524+1290 \\
& =1815
\end{aligned}
$$




\section{Toxicological Exposures - Offsite}

For the material released from the ventilation system, the offsite sumof-fractions is:

$$
\begin{aligned}
T_{\text {ventilation }} & =Q^{\prime} \text { SOF } \\
& =\left(\frac{0.00613 L}{200 \mathrm{~S}}\right)\left(94 \frac{S}{L}\right) \\
& =2.88 \times 10^{-3}
\end{aligned}
$$

where $200 \mathrm{~s}$ is the duration of the release.

Similarly for the material released from the deflagration.

$$
\begin{aligned}
T_{\text {deflagration }} & =Q^{\prime} S O F \\
& =\left(\frac{2.62 \mathrm{~L}}{200 \mathrm{~S}}\right)\left(94 \frac{\mathrm{S}}{L}\right) \\
& =1.23
\end{aligned}
$$

For the material pre-existing in the headspace, the offsite sum-offractions is:

$$
\begin{aligned}
T_{\text {headspace }} & =Q^{\prime} \text { SOF } \\
& =\left(\frac{3350 \mathrm{~m}^{3}}{200 \mathrm{~s}}\right)\left(6.6 \times 10^{-2} \frac{\mathrm{s}}{\mathrm{m}^{3}}\right) \\
& =1.106
\end{aligned}
$$

where $3350 \mathrm{~m}^{3}$ represents the volume of the headspace in single-shell tank 241-A-103.

Thus, the total toxicological exposure to the offsite receptor is

$$
\begin{aligned}
T & =T_{\text {ventilation }}+T_{\text {deflagration }}+T_{\text {beadspace }} \\
& =2.88 \times 10^{-3}+1.23+1.11 \\
& =2.3
\end{aligned}
$$




\section{REFERENCES}

Cowley, W. L., 1996, Development of Radiological Concentration and Unit Liter Doses for Tank Waste Remediation System Final Safety

Analysis Report Radiological Consequence Calculations, WHC-SD-WMSARR-037, Rev. 0, Westinghouse Hanford Company, Richland, Washington.

LANL, 1995, A Safety Assessment for Proposed Pump Mixer Operations to Mitigate Episodic Gas Releases in Tank 241-SY-101: Hanford Site, Richland, Washington, LA-UR-92-3196, Rev. 14, Los Alamos National Laboratory, Los Alamos, New Mexico.

LANL, 1996, A Safety Assessment for Salt Well Jet Pumping Operations in Tank 241-A-101: Hanford Site, Richland, Washington, WHC-SD-WMSAD-036, Rev. 0, Los Alamos National Laboratory, Los Alamos, New Mexico.

Van Keuren, J. C., and A. V. Savino, 1996, Tank Waste Compositions and Atmospheric Dispersion Coefficients for use in Safety Analys is Consequence Assessments, WHC-SD-WM-SARR-016, Rev. 2, West inghouse Hanford Company, Richland, Washington.

Van Keuren, J. C., 1996, Toxic Chemical Considerations for Tank Farm Releases, WHC-SD-WM-SARR-011, Rev. 2, Westinghouse Hanford Company, Richland, Washington.

Van Vleet, R. J., 1996, Waste Tank Ventilation System Waste Material Accumulations, WHC-SD-WM-CN-054, Rev. 0, Westinghouse Hanford Company, Richland, Washington. 


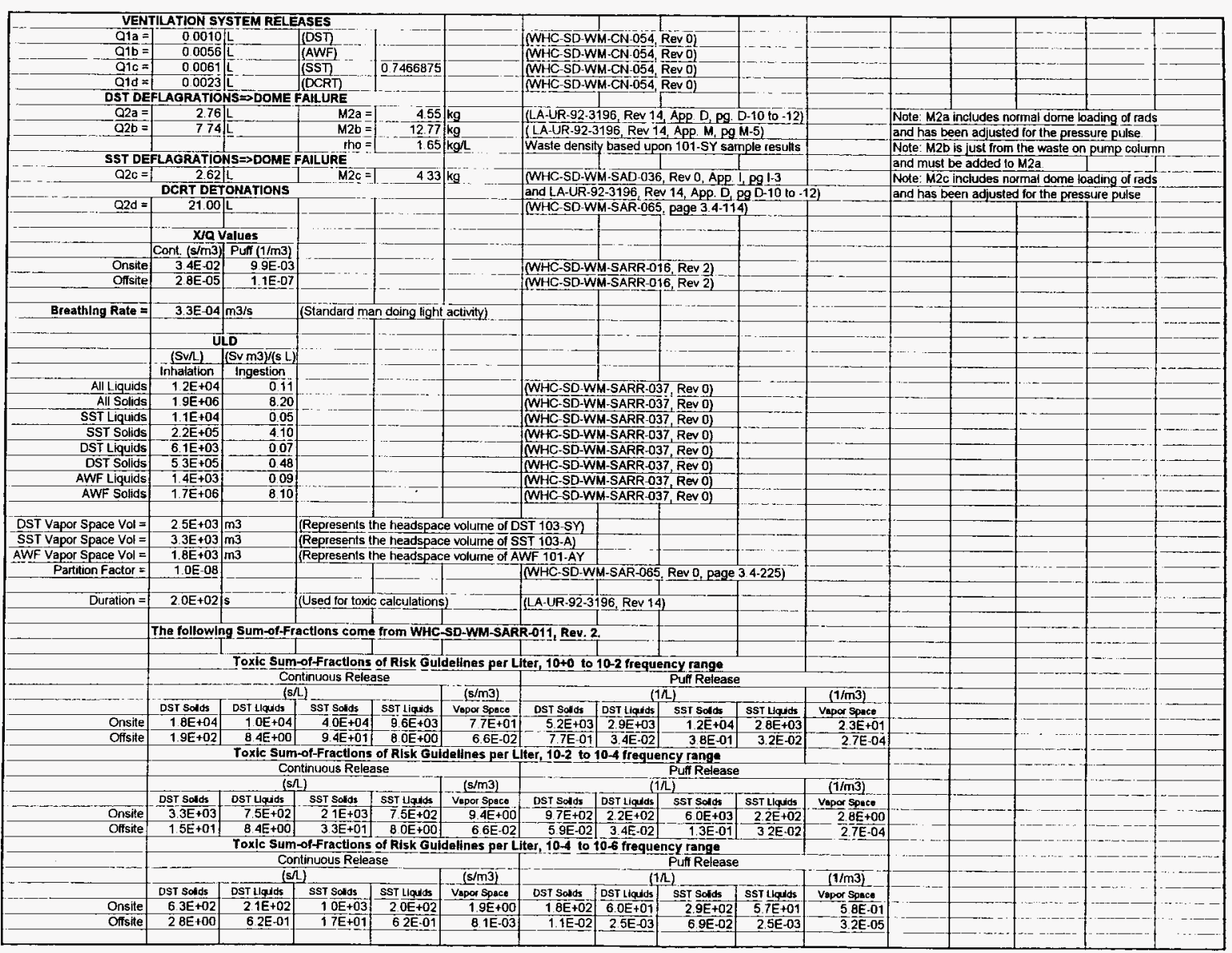




\begin{tabular}{|c|c|c|c|c|c|c|c|c|c|c|c|c|c|c|c|}
\hline \multirow{2}{*}{, } & 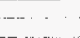 & $\cdots$ & $\cdot \cdot----$ & $\begin{array}{cc}-\cdots & \cdots \\
\end{array}$ & $\cdots-$ & $--\cdots+\cdots$ & \multirow[t]{2}{*}{ - n n } & \multirow[t]{2}{*}{$-\ldots-\cdots$} & - & \multirow{2}{*}{ 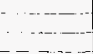 } & \multirow[t]{2}{*}{$-\cdots-$} & \multirow{2}{*}{$\longrightarrow$} & \multirow{2}{*}{$-\cdots-1$} & \multirow{2}{*}{$\begin{array}{c}\cdots--\cdots \\
-\end{array}$} & \multirow{2}{*}{ - } \\
\hline & \multicolumn{6}{|c|}{ Radiological consequences (Sv) } & & & & & & & & & \\
\hline & \multicolumn{2}{|c|}{ DST HEPA* } & \multirow{2}{*}{\multicolumn{2}{|c|}{ DST Deflagration* }} & \multicolumn{2}{|c|}{ TOTAL } & \multirow{2}{*}{$\mid-\cdots$} & & & & & & & & \\
\hline & Cont & Puff & & & Cont & Puff & & & & & & & & & \\
\hline Onstat & $67 \mathrm{E}-05$ & Not App & $16 \mathrm{E}+01$ & Not ApP & $16 \bar{E}+01$ & Not ApP & Assumes $\mathrm{D}$ & DST liguids. & & & & & & & \\
\hline Offsite & $58 E 08$ & Not App & $14 \mathrm{E}-02$ & Not ĀpP & $14 \mathrm{E} .02$ & Not App & & & & & & & & - - . & - \\
\hline & $\cdots \bar{n}$ & & & & 10 & & & & & & & & & -1 & $\cdots$ \\
\hline & DST & EPA* & DST Pump & Ejection*- & 이 & AL & & $--\ldots$ & 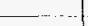 & 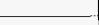 & & & & $\ldots \ldots$ & \\
\hline Onsite & $\frac{\text { Cont }}{6.7 E-05}$ & Not App & $\frac{\text { Cont. }}{6.3 \mathrm{E}+01}$ & $\begin{array}{l}\text { Puff } \\
\text { Not App. }\end{array}$ & $\frac{\operatorname{com}}{6.3 \mathrm{E}+01}$ & Not App & ${ }^{*}$ Assumes D & DST hiquids & & & - & & & - & \\
\hline Offsite & $5.8 \mathrm{E}-08$ & Not ApP & $5.2 \mathrm{E}-02$ & Not ApP & $52 E-02$ & NoI APP & & & & & & & & & \\
\hline & & & & & & & & & & & & & & & \\
\hline & AWF & IEPA* & AWF Defla & gration & TOT & $\overline{A L}$ & & & 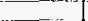 & & & & 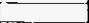 & 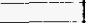 & \\
\hline & Cont & Puff & Cont & Putit & Cont & Puff & & & & & & & & & \\
\hline Onsite & $8.9 \mathrm{E}-05$ & Not App. & $5.3 \mathrm{E}+01$ & Not App. & $5.3 E+01$ & Not App & - Assumes A & AWF liquids & & & & & & & \\
\hline Offsite & $8.8 \mathrm{E}-0 \mathrm{\theta}$ & Not App & 4.4E-02 & Not App & $4.4 E-02$ & Not $A D P$. & & & & & & & & $\ldots$ & \\
\hline & & & & & & & & & & & - & & & & - \\
\hline & SSTI & IEPA* & SST Defla & gration & TOI & AL & & & & & & & & _ & 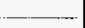 \\
\hline Onsite & Cont & Pot & $\frac{\text { Cont. }}{6.5 E+00}$ & Puff & Cont & Puff & & & & & & & & & \\
\hline Offsite & $\frac{1.5 \mathrm{E}-02}{1.3 \mathrm{E}-05}$ & Not App. & $-\frac{6 E+00}{5.7 E-03}$ & Not App & $\frac{0}{5} \cdot 7 \mathrm{E} \cdot 03$ & Not App & "Assumes & SST solids & & & & & & $\ldots \ldots$ & - \\
\hline & & & $3.045 \mathrm{E}-04$ & $5393 \mathrm{E}-03$ & & & & & & & & & & & \\
\hline & DCRT & HEPA" & DCRT Det & onation & TOI & AL & & & & & & & & & \\
\hline & Cont. & Puff & Cant. & Puif & Cont. & Puff & Assumes & SST liquides & & & & & & & \\
\hline Onsite & $2.8 E-04$ & Not APP. & $19 E+01$ & Not App & $19 E+01$ & Not App. & Assurnes & $2 / 3$ SST liquid & os \& $1 / 3 S S T$ & [solids & & & & & \\
\hline Offsite & $2.4 E-07$ & Not $\overline{A P D}$ & 1.7E-02 & Not ApP & $17 E-02$ & Not APP. & & & & & & & & & \\
\hline & & & & 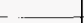 & -1 & & & & & & & & & D. & $-\ldots$ \\
\hline & & & & & & & & & 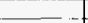 & & & & $\ldots$ & & 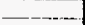 \\
\hline & $-\ldots$ & & - - - - - - & - - - & & 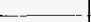 & & & 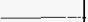 & & 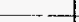 & & & $\ldots$ & _ \\
\hline$\cdots--$ & $\ldots$ & $\ldots$ & - & & - & & $\ldots$ & & & & & & & .ـ_ـ & \\
\hline Tox & cological s & umnoffracti & ons of Risk $\mathrm{G}$ & uidelines 1 & b+o to $10-2 \mathrm{ft}$ & quency r & & & & & & & & $\ldots$ & \\
\hline & DSTI & IEPA* & DST Deflia & gration & DST Vap & or Space & & DTAL & & & & & & & \\
\hline & Cont & Puif & Cont. & Puff & Cont & Puit & Cont & Punf & & & & & & & \\
\hline Onsite & $4.9 E-02$ & $2.8 \mathrm{E}+00$ & $1.4 E+02$ & $8.0 E+03$ & $96 \mathrm{E}+02$ & $5 . \overline{8 E}+04$ & $11 E+03$ & $6.5 \mathrm{E}+04$ & Assumes D & ST liguids & & & & & \\
\hline Ofisite, & $4.1 E .05$ & $3.3 E-05$ & $1.2 \mathrm{E} \cdot 0 !$ & $9.4 E-02$ & $8.3 E-01$ & $6.8 E-01$ & 94 이 & $7.7 \mathrm{E}-01$ & & & & & & $-\cdots-$ & \\
\hline - & & IEPA" & & & & & & DIAY & & & & & & $\ldots$ & \\
\hline & DSII & IEPA* & DSt Pump & Ejection" & DST Vap & I Space & To & PTAL & & & & & & & \\
\hline Onsite & $4.9 E-02$ & $28 \mathrm{E}+00$ & $5.2 \mathrm{E}+02$ & $3.0 \mathrm{E}+04$ & $9.6 \mathrm{E}+02$ & $5.2 \mathrm{E}+04$ & $1.5 E+03$ & $\frac{\text { Puil }}{8.8 E+04}$ & Assumes & IST liquids & & & & & \\
\hline Offsite & 4.1E-05 & $3.3 \mathrm{E}-05$ & $44 \mathrm{E} \cdot 01$ & $36 E-01$ & $8.3 \bar{E}-0 \bar{t}$ & $68 \mathrm{BE}-01$ & $13 E+00$ & $1 \overline{0 E}+\infty 0$ & & & & & & & \\
\hline & & & & & & & & & & & & & & & \\
\hline & AWF & $\overline{E E P A^{*}}$ & AWWF Defla & gration" & AWF Vap & or Space & & DTAL & & & & & & & - \\
\hline & Cont & Puff & Gont. & Puff & Cont & Puff & Cont & Puf́ & & & & & & & \\
\hline Onsite & $2.8 \mathrm{E}-01$ & $1.6 \mathrm{E}+01$ & $1.4 E+02$ & $8.0 E+03$ & $6.8 \mathrm{E}+02$ & $4.0 E+04$ & $8.2 E+02$ & $48 E+04$ & Assumes A & WF liquids & & & & & \\
\hline Ofsite & $2.4 E-04$ & $19 E-04$ & $1.2 \mathrm{E}-01$ & 94502 & $58 \mathrm{E}-01$ & $47 \mathrm{7E}-01$ & T.0E-01 & $5.7 E 01$ & & & & & & & \\
\hline & & & & & & & & & & & & & & $\ldots$ & \\
\hline & SSTH & EPPA* & SST Defla & gration* & SST Vap & Space & & DTAL & & & & & & & . \\
\hline Onsite & Cont & Puff & Cont. & Pulf: & Cont & Puff & Cont & Puf & & & & & & & 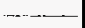 \\
\hline Offsite & $\frac{1.2 \mathrm{E}+00}{2.9 \mathrm{E}-0.3}$ & $\frac{7 E+01}{2.3 E-03}$ & $\frac{52 E+02}{1.2 E+00}$ & $-\frac{3}{10}, 0+04$ & $\frac{1}{1} \frac{3 E+03}{1 E+00}$ & $\begin{array}{l}7.7 E+04 \\
9.0 E-01\end{array}$ & $\frac{18 \mathrm{E}+03}{2 \cdot 3 \mathrm{E}+00}$ & $\frac{11}{1.9 E+05}$ & "Assumes S & ST solids & & & & & - \\
\hline & & & & & & & & & & & & & & & \\
\hline & DCRT & HEPA* & DCRT Detc & ination" & Tor & $\overline{A L}$ & & & & & & & & (5) & \\
\hline & Cont & Puff & Cont. & Puff & Cont. & Puff & & & "Assumes $S$ & ST liquids & & & & & \\
\hline Onsite & 1. $1 \mathrm{E}-01$ & $6.4 E+00$ & $2.1 E+03$ & $1.2 \mathrm{E}+05$ & $21 \mathrm{E}+03$ & $1.2 E+05$ & & & Acsumes & $2 \sqrt{3}$ SST liquids & $81 / 3 \bar{S}$ & solids & & & \\
\hline Offsite & $9.1 \mathrm{E}-05$ & $7-3 \bar{E}-05$ & $3 . B E+00$ & $31 \mathrm{E}+00$ & $3.8 \bar{E}+\overline{00}$ & $3.1 \mathrm{E}+00$ & & & & & & & & & \\
\hline & & & & & & & & & & & & & & $\ldots \ldots$ & $-\ldots$ \\
\hline & & & & & & & & & & & & & & $\ldots$ & - \\
\hline & & & & & & & & & & & & & & & \\
\hline
\end{tabular}




\begin{tabular}{|c|c|c|c|c|c|c|c|c|c|c|c|c|c|c|c|}
\hline \multicolumn{9}{|c|}{ Toxicological Sum-of-Fractions of Risk Guldelines, $10-2$ to $10-4$ frequency range } & & \multirow{3}{*}{$\begin{array}{l}- \\
\cdots-\end{array}$} & \multirow{3}{*}{ - } & \multirow{3}{*}{ 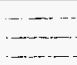 } & \multirow{3}{*}{ - } & \multirow{3}{*}{ 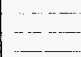 } & \multirow{3}{*}{ - . - - } \\
\hline & 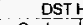 & EPA* & DST Defla & ration* & DST Vap & ISpace & To & TAL & & & & & & & \\
\hline & Cont & Puff & Cont & Puif & Cont & Puff & Cont & Puit & & & & & & & \\
\hline Onsite & $37 \mathrm{E}-03$ & $22 \mathrm{E}-01$ & $1.0 \mathrm{E}+01$ & $6 \frac{1 E+02}{1 E}$ & $12 \frac{\mathrm{E}+02}{2}$ & $70 E+03$ & $13 \mathrm{E}+02$ & $76 E+03$ & "Assumes D & STi liquids & & & & & \\
\hline ofsite & $41 \bar{E}-05$ & $3.3 E-05$ & $12 E-01$ & $\overline{9}-4 E-02$ & 8 3E-01 & 6 틈 & $9 \overline{4 E}-\overline{01}$ & $7.7 E-01$ & & & & & & & \\
\hline & DSTH & $\overline{A E A^{*}}$ & DSTT Pump & jection" & OST Vap & ispace & ro & TAL & & & & & & -- & \\
\hline & Cont & Puff & Cont. & Fuff & Cont. & Pufi & Cont & Punf & & & & & & - & \\
\hline Onsite & $37 \mathrm{E}-03$ & $2.2 \mathrm{E}-01$ & $3.9 E+01$ & $23 \overline{3}+03$ & $1.2 \mathrm{E}+02$ & $7.0 \mathrm{E}+03$ & $16 \mathrm{E}+02$ & $93 \mathrm{E}+03$ & "Assumes $D$ & ST tiguids & & & & & \\
\hline Offsite & $41 \bar{E}-\overline{0} 5$ & $33 \bar{E}-\overline{0} 5$ & $4.4 E-01$ & $36 \mathrm{EE}-01$ & $83 \mathrm{E}-01$ & $68 E-01$ & $13 \overline{3}+00$ & $10 \mathrm{E}+00$ & & & & & & $\ldots$ & $\ldots$ \\
\hline & & & & & & & & & & & & & & & .... \\
\hline & AWF & HEPA* & AWF Defla & gration" & AWF Vap & of Space & TO & TAL & & & & & & - & \\
\hline & Cont & Puff & Cont & Puff & Cont & Puff & Cont & Puff & & & & & & & \\
\hline Onsitte & $2.1 \mathrm{E}-02$ & $12 E+00$ & $1.0 \mathrm{E}+01$ & $6.1 \mathrm{E}+02$. & $8.3 \mathrm{E}+0 \mathrm{i}$ & $49 \mathrm{E}+03$ & $-93 E+01$ & $5.5 E+03$ & $\ddot{A}$ Assumes $A$ & WWF liquids & & & & & \\
\hline Offsite & $24 E-04$ & $1.9 \mathrm{E}-04$ & $1.2 E \cdot 01$ & $94 \mathrm{E}-02$ & $5.8 E-01$ & $4.7 E-01$ & $70 \mathrm{E}-01$ & $\overline{5} \overline{7 E}-01$ & & & & & & & \\
\hline & SSTH & EPA* & SST Defla & rration" & SST Vapd & I Space & & TAI & & & & & & 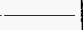 & \\
\hline & Cont. & Puiff & Cont & Puff & Cont. & $\frac{1 \text { space }}{\text { Puff }}$ & Cont & Pul & & & & & & & \\
\hline Onsite & $64 \mathrm{AE}-02$ & $37 \mathrm{E}+01$ & $28 \mathrm{E}+01$ & $16 \bar{E}+0 \overline{4}$ & $1.6 \bar{E}+02$ & $9.4 E+03$ & $19 \mathrm{E}+02$ & $25 E+04$ & Assumes S & SST solids & & & & & 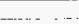 \\
\hline Offsite: & $10 \mathrm{E}-03$ & $80 E-04$ & $4 \overline{3 E} \cdot 01$ & $34 \mathrm{E} \cdot 01$ & $1.1 \mathrm{E}+00$ & $90 E .01$ & $1.5 \bar{E}+00$ & $12 E+00$ & & & & & & & $\ldots$ \\
\hline & DCRT & & DCRT Detc & nation"* & TOT & AL & & & & & $-\ldots$. & & & - & \\
\hline & Cont & PUAf & Cont & Puff & Cont. & AL Puff & & 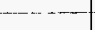 & Assumes S & SST liquids & & & & $--\cdot$ & \\
\hline Onsite & $8.5 \mathrm{E}-03$ & $5.0 E-01$ & $13 \mathrm{E}+02$ & $45 \mathrm{E}+04$ & $13 E+02$ & $4.5 \mathrm{E}+04$ & & & "Assumes & $\overline{2 / 3} \overline{S S T}$ liquid & $81 / 3 \mathrm{SST}$ & solids & & & 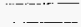 \\
\hline Offsite & $9.1 \mathrm{E}-05$ & 7.3트-05 & $1 . \bar{E}+00$ & $14 E+00$ & $17 \mathrm{E}+00$ & $1 . \overline{4} \mathrm{E}+0 \overline{0}$ & & & & & & & & . & $-\ldots$ \\
\hline & & & & & & & & & & 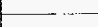 & & & & - - . - - & - \\
\hline & & & & & & & & & & & & & & - & $\cdots$ \\
\hline & & & & $\cdots$ & & & & & & & & & & $\cdots$ & - \\
\hline & & & - & & & - & & & & & 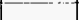 & & & -- & - - \\
\hline Tox & cologlcal s & um-of-Fracth & ons of Risk & uldelines, 1 & $0-4$ to $10-6 \mathrm{fr}$ & quency rang & & & & & & & & $-\ldots$ & - \\
\hline & DST & EPA* & DSIDefla & gration & DST Vap & is Space & TO & TAL & & & & & & & \\
\hline & Cont. & Puff: & cont & Puff & Cont. & Puff & Cont & Puff & & & & & & & _._........ \\
\hline Onsite & $1.0 \mathrm{E} .03$ & $5.9 \mathrm{E}-02$ & $29 \bar{E}+00$ & $1.7 \mathrm{E}+02$ & $2.4 E+01$ & $1.5 E+03$ & $27 E+01$ & $16 \mathrm{E}+03$ & Assumes I & STT hquids & & & & & \\
\hline Ofisite & $30 \mathrm{OE}-06$ & $24 \mathrm{E}-06$ & $8.5 \mathrm{E}-03$ & $6.9 E-03$ & $10 E-01$ & $80 \mathrm{E}-02$ & 1. $1 \mathrm{E}-01$ & $8 \overline{7 E}-\overline{0} 2$ & & & & & & $\ldots$ & \\
\hline & & & & & & & & & & & & & & $\ldots \ldots$ & \\
\hline & DST F & IEPA* & DST Pump & Ejection" & DST Vap & ispace & To & TAL & & & & & & -... & $\ldots$ \\
\hline & Cont & Puif & Cont & Puff & Cont & Puff & Cont & Puff & & & & & & & $\ldots$ \\
\hline Onsite & $1.0 \mathrm{E}-03$ & $5 \overline{9 E-02}$ & $11 E+01$ & $63 E+02$ & $2.4 \mathrm{E}+01$ & 1. $5 E+03$ & $3.5 E+01$ & $2.1 E+03$ & Assumes & ST liquids & & & & & ..... \\
\hline Ofisite & $30 \mathrm{OE}$ & 2.4E-06 & $3.3 \mathrm{E}-02$ & $26 \mathrm{E}-02$ & 1.0E-01 & $8.0 \mathrm{E}-02$ & $13 \mathrm{E}-0 \mathrm{i}$ & $1 \overline{1 E}-01$ & & & & & & - & \\
\hline & $A W F$ & IEPA* & AWF Defle & gration" & AWF Vap & r Space & & TAL & & & & & & 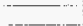 & \\
\hline & Cont. & Puff & Cont & Pulf & Cont. & Püt & Conl & Pulf & & & & & & & \\
\hline Onsite & 5 GE-03 & $34 \mathrm{E}-01$ & $29 E+00$ & $17 \bar{E}+02$ & $1.7 \mathrm{E}+01$ & $1.0 E+03$ & $20 E+01$ & $12 E+03$ & Assumes $A$ & WF liquids & & & & & \\
\hline Otsilte & 1.7E-0S & $14 \mathrm{E}-05$ & $85 E-0 \overline{3}$ & $69 \mathrm{gE}-03$ & $7.1 E-02$ & $5.6 E-02$ & B.0E.02 & $6.3 \mathrm{E}-02$ & & & & & & ---- & \\
\hline & & & & & & & & & & & & & & & \\
\hline & SSTI & EPA* & SST Defla & gration* & SST Vap & is Space & & DTAL & & & & & & - - - & \\
\hline & Cont. & Puff & Cont & Puff & Cont & Puff & Cont. & Puff & & & & & & 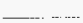 & \\
\hline Onsite & $3.1 \mathrm{E}-02$ & $1.8 E+00$ & $13 E+01$ & $7.6 \mathrm{E}+02$ & $32 E+01$ & $19 E+03$ & $4.5 E+01$ & $2.7 E+03$ & "Assumes & ST solids & & & & & \\
\hline Ofisite & $5.2 \mathrm{E}-04$ & $4.2 \mathrm{E}-04$ & $22 \mathrm{E} 01$ & $18 \mathrm{E}-01$ & 1.4E-01 & $11 \overline{01}$ & $36 \mathrm{E}-01$ & $2.9 E-01$ & & & & & & - & \\
\hline & & & & & & $\overrightarrow{A I}$ & & & & & $\ldots$ & & & & 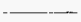 \\
\hline & DCRT & IEPA* & DCRT Det & nation:" & & AL & & & & & 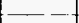 & & & & \\
\hline & Cont. & Puff & Cont & Puff & Cont & Puff & & & *Assumes $\mathrm{S}$ & \$ST liguids & & & $\therefore$ & $\ldots-\cdots$ & --- \\
\hline Onsite & 2. 3E -03 & 1.3E-01 & $49 \mathrm{gE}+01$ & $2.8 E+03$ & $49 \mathrm{E}+01$ & $\begin{array}{r}2.8 \mathrm{E}+03 \\
5.5-01\end{array}$ & & & Assumes & $2 / 3$ sST liquidd & $\& 1 / 3$ SS & solids & & $-\cdots$ & - \\
\hline Offsitel & $71 \mathrm{E}-06$ & $5.7 E-06]$ & $63 \mathrm{E}-01$ & 5.1E-01] & $6.3 \mathrm{E}-01$ & 5.1E-01] & & & & & & & & & \\
\hline
\end{tabular}




\section{CHECKLIST FOR PEER REVIEW}

Document Reviewed: WHC-SD-WM-CN-055, Rev. 0, Flammable Gas Deflagration Consequence Calculations for the Tank Waste Remediation System Basis for Interim operation

Scope of Review: Entire document.

$\frac{\text { Yes No NA }}{[][][X] *}$

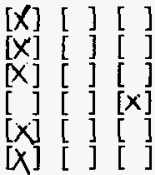

$[x][][]$

$[x][$ ] [ ]

$[X][][]$

$[x]\left[\begin{array}{l}{[x]} \\ {[x]}\end{array}\right]$

$[X][][]$

$[\mathrm{C}][\mathrm{X}]$ [X] [ ] [ ]

$[x][][]$

[ ] [ ] [X]

[ ] [X] *

Previous reviews complete and cover analysis, up to scope of this review, with no gaps.

Problem completely defined.

Accident scenarios developed in a clear and logical manner. Necessary assumptions explicitly stated and supported. Computer codes and data files documented. Data used in calculations explicitly stated in document.

Data checked for consistency with original source information as applicable.

Mathematical derivations checked including dimensional consistency of resu1ts.

Models appropriate and used within range of validity or use outside range of established validity justified.

Hand calculations checked for errors. Spreadsheet results should be treated exactly the same as hand calculations.

Software input correct and consistent with document reviewed.

Software output consistent with input and with results reported in document reviewed.

Limits/criteria/guidelines applied to analysis results are appropriate and referenced. Limits/criteria/guidelines checked against references.

Safety margins consistent with good engineering practices.

Conclusions consistent with analytical results and applicable limits.

Results and conclusions address all points required in the problem statement.

Format consistent with appropriate NRC Regulatory Guide or other standards

Review calculations, comments, and/or notes are attached.

[X] [ ] [ ] Document approved.

C. H. Huang $e$ H Huancy Reviewer (Printed Name and Signatyfe) $8 / 8 / 1996$ Date

* Any calculations, comments, or notes generated as part of this review should be signed, dated and attached to this checklist. Such material should be labeled and recorded in such a manner as to be intelligible to a technically qualified third party. 